Catherine Van Poznak and Daniel F. Hayes, University of Michigan Comprehensive Cancer Center, Ann Arbor, Ml; Mark R. Somerfield, American Society of Clinical Oncology, Alexandria, VA; Robert C. Bast, Ana M. Gonzalez-Angulo, and William F. Symmans, University of Texas MD Anderson Cancer Center, Houston; Robert G. Mennel, Texas Oncology, Dallas, TX; Massimo Cristofanilli, Thomas Jefferson University-Kimmel Cancer Center, Philadelphia, PA; Matthew P. Goetz and Minetta C. Liu, Mayo Clinic, Rochester, MN; David G. Hicks, University of Rochester Medical Center, Rochester, NY; Elizabeth G. Hill, Medical University of South Carolina, Hollings Cancer Center, Charleston, SC; Wanda Lucas, Georgetown University, Washington, DC; Ingrid A. Mayer, Vanderbilt University Medical Center and Vanderbilt-Ingram Cancer Center, Nashville, TN; and Lyndsay N. Harris, Seidman Cancer Center, Case Western Reserve University, Cleveland, $\mathrm{OH}$

Published online ahead of print at www.jco.org on July 20, 2015

Clinical Practice Guideline Committee approval: January 21, 2015

Editor's note: This American Society of Clinical Oncology clinical practice guideline provides recommendations, with comprehensive review and analyses of the relevant literature for each recommendation. Additional information, including a Data Supplement with additional evidence tables, a Methodology Supplement, slide sets, clinical tools and resources, and links to patient information at http://Mww .cancer.net is available at http://www.asco org/guidelines/metastaticbreastmarkers.

Authors' disclosures of potential conflicts of interest are found in the article online at www.jco.org. Author contributions are found at the end of this article.

Corresponding author: American Society of Clinical Oncology, 2318 Mill Rd, Suite 800, Alexandria, VA 22314; e-mail: guidelines@asco.org.

(C) 2015 by American Society of Clinical Oncology

0732-183X/15/3324w-2695w/\$20.00

DOI: $10.1200 / J C O .2015 .61 .1459$

\title{
Use of Biomarkers to Guide Decisions on Systemic Therapy for Women With Metastatic Breast Cancer: American Society of Clinical Oncology Clinical Practice Guideline
}

Catherine Van Poznak, Mark R. Somerfield, Robert C. Bast, Massimo Cristofanilli, Matthew P. Goetz, Ana M. Gonzalez-Angulo, David G. Hicks, Elizabeth G. Hill, Minetta C. Liu, Wanda Lucas, Ingrid A. Mayer, Robert G. Mennel, William F. Symmans, Daniel F. Hayes, and Lyndsay N. Harris

\section{$\begin{array}{llllllll}\text { A } & \text { B } & S & \mathbf{T} & \mathbf{R} & \mathbf{A} & \mathbf{C} & \mathbf{T}\end{array}$}

\section{Purpose}

To provide recommendations on the appropriate use of breast tumor biomarker assay results to guide decisions on systemic therapy for metastatic breast cancer.

\section{Methods}

A literature search and prospectively defined study selection identified systematic reviews, meta-analyses, randomized controlled trials (RCTs), prospective-retrospective studies, and prospective comparative observational studies published from 2006 through September 2014.

\section{Results}

The literature search revealed 17 articles that met criteria for further review: 11 studies reporting discordances between primary tumors and metastases in expression of hormone receptors or human epidermal growth factor receptor 2 (HER2), one RCT that addressed the use of a biomarker to decide whether to change or continue a treatment regimen, and five prospective-retrospective studies that evaluated the clinical utility of biomarkers.

\section{Recommendations}

In patients with accessible metastases, biopsy for confirmation of disease process and retesting of estrogen receptor, progesterone receptor, and HER2 status should be offered, but evidence is lacking to determine whether changing anticancer treatment on the basis of change in receptor status affects clinical outcomes. With discordance of results between primary and metastatic tissues, the Panel consensus is to use preferentially the estrogen receptor, progesterone receptor, and HER2 status of the metastasis to direct therapy if supported by the clinical scenario and patient's goals for care. Carcinoembryonic antigen, cancer antigen 15-3, and cancer antigen 27-29 may be used as adjunctive assessments, but not alone, to contribute to decisions regarding therapy. Recommendations for tumor rebiopsy and use of circulating tumor markers are based on clinical experience and Panel informal consensus in the absence of studies designed to evaluate the clinical utility of the markers. As such, it is also reasonable for clinicians to not use these markers as adjunctive assessments.

\section{J Clin Oncol 33:2695-2704. (C) 2015 by American Society of Clinical Oncology}

\section{INTRODUCTION}

The purpose of this guideline is to provide evidencebased recommendations to practicing oncologists and other stakeholders on the appropriate use of results from assays of breast tumor biomarkers to guide or influence decisions on systemic therapy in women with metastatic breast cancer. A previous update of the American Society of Clinical Oncology (ASCO) guideline on use of tumor markers in breast cancer ${ }^{1}$ considered all indications or uses for biomarker assay results. Subsequently, ASCO collaborated with the College of American $\mathrm{Pa}$ - thologists to publish and then update a guideline on testing for human epidermal growth factor receptor 2 (HER2) ${ }^{2,3}$ and another on testing for endocrine (estrogen [ER] and progesterone [PR]) receptors. ${ }^{4}$ To facilitate future updates in a rapidly developing and expanding field, the ASCO Breast Cancer Guideline Advisory Group and Clinical Practice Guideline Committee determined that guidelines on additional breast cancer biomarkers should focus on single uses or indications, and selected the use of biomarkers to guide or influence decisions on systemic therapy as the topic of highest priority. 


\section{Recommendations for Use of Biomarkers to Guide Decisions on Systemic Therapy for Women With Metastatic Breast Cancer: ASCO Clinical Practice Guideline}

\section{Guideline Questions}

1. Should metastases be biopsied or otherwise sampled to test for changes from the primary tumor with respect to ER, PR, or HER2 status?

2. For women with metastatic breast cancer and with known ER, PR, and HER2 status, which additional tumor markers have demonstrated clinical utility to initiate systemic therapy or direct selection of a new systemic therapy regimen?

3. For women with metastatic breast cancer and with known ER, PR, and HER2 status, which additional tumor markers have demonstrated clinical utility to guide decisions on switching to a different drug or regimen or discontinuing treatment?

4. For biomarkers shown to have clinical utility to guide decisions on systemic therapy for metastatic disease in questions 2 and 3, what are the appropriate assays, timing, and frequency of measurement?

\section{Target Population}

Women with metastatic breast cancer being considered for systemic therapy or for changes in the drug or regimen they are receiving.

\section{Target Audience}

Any physician caring for patients with breast cancer, including medical, surgical, and radiation oncologists; oncology nurses and physician assistants, pathologists, and patients.

\section{Methods}

An Expert Panel was convened to develop clinical practice guideline recommendations based on a systematic review of the medical literature.

\section{Key Points}

- Patients with accessible, newly diagnosed metastases from primary breast cancer should be offered biopsy for confirmation of disease process and testing of ER, PR, and HER2 status. They should also be informed that if discordances are found, evidence is lacking to determine whether outcomes are better with treatment regimens based on receptor status in the metastases or the primary tumor. With discordance of results between primary and metastatic tissues, the Panel consensus is to preferentially use the ER, PR, and HER2 status from the metastasis to direct therapy, if supported by the clinical scenario and the patient's goals for care. (Type: evidence based for biomarker change from primary to metastasis, but no evidence to address systemic therapy choices affecting health outcome when biomarker change occurs. Evidence quality: insufficient. Strength of recommendation: moderate.)

- Decisions on initiating systemic therapy for metastatic breast cancer should be based on clinical evaluation, judgment, and patient preferences. There is no evidence at this time that initiating therapy solely on the basis of biomarker results beyond those of ER, PR, and HER2 improves health outcomes. (Type: evidence based. Evidence quality: low. Strength of recommendation: moderate.)

- Recommendations for tissue biomarkers: In patients already receiving systemic therapy for metastatic breast cancer, decisions on changing to a new drug or regimen or discontinuing treatment should be based on clinical evaluation, judgment of disease progression or response, and the patient's goals for care. There is no evidence at this time that changing therapy based solely on biomarker results beyond ER, PR, and HER2 improves health outcomes, quality of life, or cost effectiveness. (Type: evidence based. Evidence quality: low. Strength of recommendation: moderate.)

- Recommendations for circulating tumor markers: In patients already receiving systemic therapy for metastatic breast cancer, decisions on changing to a new drug or regimen or discontinuing treatment should be based on clinical evaluation, judgment of disease progression or response, and the patient's goals for care. There is no evidence at this time that changing therapy based solely on circulating biomarker results improves health outcomes, quality of life, or cost effectiveness. (Type: evidence based. Evidence quality: intermediate. Strength of recommendation: moderate.) (continued on following page) 
- CEA, CA 15-3, and CA 27-29 may be used as adjunctive assessments to contribute to decisions regarding therapy for metastatic breast cancer. Data are insufficient to recommend use of CEA, CA 15-3, and CA 27-29 alone for monitoring response to treatment. The recommendation for use is based on clinical experience and Panel informal consensus in the absence of studies designed to evaluate the clinical utility of the markers. As such, it is also reasonable for clinicians to not use these markers as adjunctive assessments. (Type: informal consensus. Evidence quality: insufficient. Strength of recommendation: moderate.)

ASCO believes that cancer clinical trials are vital to inform medical decisions and improve cancer care and that all patients should have the opportunity to participate.

Many reviews and commentaries have discussed the nature of evidence required to support conclusions that using biomarker assay results to guide or influence treatment decisions improves health outcomes for patients with a malignancy. ${ }^{5-11}$ Although nearly two decades have passed since clinical investigators began addressing these issues, ${ }^{12}$ this guideline (and the companion guideline on systemic therapy for women with early-stage breast cancer) applies in part the framework developed by the Evaluation of Genomic Applications in Practice and Prevention Working Group. ${ }^{13,14}$ In this framework, assays must demonstrate analytic validity, clinical validity, and clinical utility before they are used routinely to guide or influence treatment decisions. Data Supplement Table 1 provides definitions of these terms and evidentiary requirements to demonstrate that a specific biomarker assay is valid and clinically useful. Note that these new criteria are more rigorous than those applied in the 2007 ASCO biomarker guideline.

As discussed by Simon et al, ${ }^{11}$ study designs to test for clinical utility of tumor biomarkers may include systematic reviews or metaanalyses, randomized controlled trials (RCTs), retrospective analyses of biologic samples from patients enrolled onto already completed prospective RCTs (hereinafter referred to as prospective-retrospective studies), and comparative observational studies. Evidence on analytic and clinical validity serves as the foundation of biomarker development and is reviewed in this guideline only for biomarkers with definitive evidence of clinical utility, given that it is clinical utility that matters for patient care.

\section{CLINICAL UTILITY EVALUATION GUIDELINE}

The charge to the Panel was to evaluate evidence for clinical utility of biomarkers in breast cancer. This evaluation required the following: clear definitions of clinical utility; identification of studies that were designed, conducted, and analyzed rigorously to provide reliable evidence; and review of the results of these studies to assess evidence for clinical utility.

\section{Clinical Utility}

A biomarker-based test is judged to have clinical utility if use of the test is demonstrated to result in a favorable balance of benefits to harms. ${ }^{1-3}$ If clinical care options result in similar patient survival, biomarker tests may still have clinical utility if they direct care to options that result in improved quality of life (eg, less toxicity or inconvenience) or lower cost. A new biomarker test must be shown to contribute clinically useful information beyond that already provided by standardly used clinical or pathologic indicators, unless the new test can provide equivalent information at a lower cost, less invasively, or with less inconvenience or risk.

\section{Reliable Evidence}

The most reliable evidence can be obtained from well-designed and well-executed studies. Requirements for inclusion of a study in the review stipulated that the study was either a fully prospective study (ie, specifically designed to answer the biomarker test-related question and conducted prospectively) or that the study met the criteria for a prospective-retrospective study. ${ }^{11}$ Prospective studies are classified as category A evidence and prospective-retrospective studies as category $\mathrm{B}$ evidence, according to the classification provided by Simon et al, ${ }^{11}$ whereas the cumulative evidence for a biomarker test is assessed for determination of an overall level of evidence. ${ }^{11}$ For example, a biomarker test is considered to have reached level IB evidence for clinical utility if it meets the criteria listed in Table 1.

Table 1. Requirements for Marker-Based Test to Reach Level IB Evidence of Clinical Utility Based on Prospective-Retrospective Studies

$$
\text { Requirement }
$$

Adequate amounts of archived specimen must be available from enough patients from prospective trial (which for predictive factors should generally have randomized design) for analyses to have adequate statistical power and for patients included in evaluation to be clearly representative of patients in trial

Marker-based test should be analytically and preanalytically validated for use with archived specimens

Plan for marker evaluation should be completely specified in writing before performance of marker assays on archived specimens and should be focused on evaluation of single, completely defined marker-based test

Results from archived specimens should be validated using specimens from $\geq$ one similar, but separate, studies

NOTE. Adapted with permission. ${ }^{11}$ 


\section{Additional Considerations}

The Panel reached a final recommendation for each biomarker test evaluated on the basis of application of the specified levels of evidence criteria. However, some subjective judgment was required to reach consensus on whether the observed magnitude of biomarker effect was sufficiently large to be clinically meaningful and whether the statistical strength of the evidence was sufficient. Additional challenges included the lack of information reported in many published tumor marker studies. Panel members used their expertise (clinical, laboratorial, and statistical) and personal experience to aid in reaching consensus.

\section{GUIDELINE QUESTIONS}

This clinical practice guideline addresses four overarching clinical questions:

- (1) Under what circumstances (ie, for which patients) should metastases be biopsied or otherwise sampled to test for changes from the primary tumor with respect to endocrine receptor or HER2 status?

- (2) For women with metastatic breast cancer and with known endocrine receptor and HER2 status, which additional tumor markers have demonstrated clinical utility to guide initiation of systemic therapy or direct selection of a new systemic therapy regimen? Evidence addressing this question and the resulting recommendations were considered separately for women with metastatic breast cancer that is endocrine receptor positive, HER2 positive, or negative for both endocrine receptors and HER2 (ie, triple negative). Within each of these patient subsets, evidence and recommendations were considered separately for decisions on first-line versus second- or subsequent-line systemic therapy for metastatic disease.

- (3) For women with metastatic breast cancer and with known ER, PR, and HER2 status, which additional tumor markers have demonstrated clinical utility to guide decisions on whether to switch to a different drug or regimen or discontinue treatment? Evidence and recommendations for this question were also considered separately for women with metastatic breast cancer that is endocrine receptor positive, HER2 positive, or negative for both endocrine receptors and HER2 (triple negative).

- (4) For each tumor marker shown to have clinical utility for guiding decisions on systemic therapy for metastatic disease in questions 2 or 3, what are the appropriate assays, timing, and frequency of measurement?

\section{METHODS}

\section{Guideline Development Process}

The recommendations were developed by a multidisciplinary Expert Panel (Appendix Table A1, online only) that met face to face, via teleconference, and/or via Webinar and corresponded through e-mail. All authors evaluated the evidence and contributed to development of the guideline by critically reviewing and approving the guideline recommendations. All ASCO guidelines are ultimately reviewed and approved by the Expert Panel and the ASCO Clinical Practice Guideline Committee before publication.
The Panel developed its recommendations on the basis of evidence identified through online searches of MEDLINE and the Cochrane Library (from January 2006 through September 2014, to overlap with the search end date for the 2007 guideline update on tumor markers in breast cancer) ${ }^{1}$ and Panel members' own clinical experience. See Data Supplement 5 for full details on the search string. A combined PubMed search was conducted for this guideline. Articles were selected for inclusion in the systematic review on the basis of the following criteria:

- Population: Women with metastatic breast cancer being considered for initiation of systemic therapy or for changes in the drug or regimen they are receiving, with separate subquestions and analyses on patient groups with endocrine receptor-positive disease, HER2positive disease, and triple-negative disease and on use of tumor marker assay results to guide decisions on first-line therapy and second- or subsequent-line therapy.

- For clinical questions 1 to 3, publications in English were included if they reported rigorously conducted systematic reviews (with or without meta-analyses), RCTs, retrospective biomarker analyses of samples from completed prospective RCTs, or prospective observational studies that directly compared outcomes of treatment decisions made on the basis of assay results with outcomes of treatment decisions made regardless of assay results.

- For clinical question 4, studies that directly compared performance characteristics of different assay methods or different times or frequencies of measurement were included only for markers with clinical utility demonstrated as described in questions 2 and 3.

Articles were excluded from the systematic review if they were meeting abstracts not subsequently published in peer-reviewed journals; editorials, commentaries, letters, news articles, case reports, or narrative reviews; published in a language other than English; or retrospective observational studies. The guideline recommendations were crafted, in part, using principles of the Guidelines Into Decision Support methodology. ${ }^{15}$ Ratings for the type and strength of the recommendation, evidence, and potential bias are provided with each recommendation (Methodology Supplement).

Detailed information about the methods used to develop this guideline is available in the Methodology Supplement at http://www.asco.org/guidelines/ vanity, including an overview (eg, Panel composition, development process, and revision dates) and information on the literature search and data extraction, recommendation development process, and quality assessment.

\section{Guideline Disclaimer}

The clinical practice guideline and other guidance published herein are provided by ASCO to assist providers in clinical decision making. The information herein should not be relied on as being complete or accurate, nor should it be considered as inclusive of all proper treatments or methods of care or as a statement of the standard of care. With the rapid development of scientific knowledge, new evidence may emerge between the time information is developed and when it is published or read. The information is not continually updated and may not reflect the most recent evidence. The information addresses only the topics specifically identified herein and is not applicable to other interventions, diseases, or stages of diseases. This information does not mandate any particular course of medical care. Furthermore, the information is not intended to substitute for the independent professional judgment of the treating provider, because the information does not account for individual variation among patients. Recommendations reflect high, moderate, or low confidence that the recommendation reflects the net effect of a given course of action. The use of words like "must," "must not," "should," and "should not" indicates that a course of action is recommended or not recommended for either most or many patients, but there is latitude for the treating physician to select other courses of action in individual cases. In all cases, the selected course of action should be considered by the treating provider in the context of treating the individual patient. Use of the information is voluntary. ASCO provides this information on an as-is basis and makes no warranty, express or 
implied, regarding the information. ASCO specifically disclaims any warranties of merchantability or fitness for a particular use or purpose. ASCO assumes no responsibility for any injury or damage to persons or property arising out of or related to any use of this information or for any errors or omissions.

\section{Guideline and Conflicts of Interest}

The Expert Panel was assembled in accordance with the ASCO Conflicts of Interest Management Procedures for Clinical Practice Guidelines (summarized at http://www.asco.org/rwc). Members of the Panel completed the ASCO disclosure form, which requires disclosure of financial and other interests that are relevant to the subject matter of the guideline, including relationships with commercial entities that are reasonably likely to experience direct regulatory or commercial impact as a result of promulgation of the guideline. Categories for disclosure include employment; leadership; stock or other ownership; honoraria, consulting, or advisory role; speaker's bureau; research funding; patents, royalties, or other intellectual property; expert testimony; travel, accommodations, or expenses; and other relationships. In accordance with these procedures, the majority of the members of the Panel did not disclose any such relationships. Notably, one author (D.F.H.) recused himself from deliberations and Panel votes concerning recommendations for circulating tumor cells (CTCs) because of potential conflicts of interest.

\section{LITERATURE SEARCH RESULTS}

Seventeen reports from 16 separate studies met eligibility criteria (see Data Supplements 2, 3, and 4). Eleven published articles (from 10 separate studies) reported on discordances between paired samples (from primary breast tumors and subsequent metastases) with respect to expression of ER, PR, and HER2. This set of publications (see Data Supplement 3) included three systematic reviews and metaanalyses ${ }^{16-18}$ and seven comparative observational studies. ${ }^{19-26} \mathrm{Six}$ studies addressed clinical utility, as defined in Clinical Utility section, for initiating or selecting therapy for metastatic breast cancer. The Smerage et $\mathrm{al}^{27}$ study addressed clinical utility in the context of an RCT. Five prospective-retrospective studies also addressed clinical utility. ${ }^{28-32}$

Data Supplements 3 and 4 include results of data extraction from the literature review. Data Supplement Tables 2 through 7 provide information on key characteristics of the studies and their patient populations, on study quality assessment, and on reported outcomes. None of the included studies reported on toxicity, either of biomarker testing or of systemic therapy, for patient subgroups subdivided by biomarker results. In addition, no studies reported on changes in quality-of-life outcomes attributable to biomarker testing.

\section{RECOMMENDATIONS}

\section{CLINICAL QUESTION 1}

Under what circumstances (ie, for which patients) should metastases be biopsied or otherwise sampled to test for changes from the primary tumor with respect to endocrine receptor or HER2 status?

\section{Recommendation 1}

At initial presentation of metastasis from breast cancer, it is standard of care to biopsy an accessible lesion to confirm metastatic breast cancer. When evaluating the metastatic site(s), it is important to note that the results of ER, PR, and/or HER2 status may have changed from the primary tumor, and these results may inform treatment decisions. Therefore, this Panel recommends restesting for ER, PR, and HER 2 on $\geq$ one metastasis with careful attention to assay performance, particularly for bone metastases (see Clinical Interpretation section). However, for patients with documented changes in these biomarkers, data are lacking to determine whether outcomes from systemic therapy are altered when guided by biomarker test results from the metastases. The Panel informal consensus for the management of care when there is discordance of ER, PR, or HER2 results between primary and metastatic tissues is to use the ER, PR, or HER2 status from the metastasis to direct therapy, if supported by the clinical scenario and the patient's goals for care. (Type: evidence based for biomarker change from primary to metastasis, but no evidence to demonstrate that systemic therapy choices affect health outcomes when biomarker change occurs. Evidence quality: insufficient. Strength of recommendation: moderate.)

\section{Literature Review and Analysis}

Studies on the frequency of changes in ER, PR, and HER2 expression from primary tumors to metastases were retrospective or prospective observational studies. This applied to all studies included in three systematic reviews and meta-analyses, as well as the seven original studies listed in Data Supplement 3. None of the studies reported on treatments used for metastatic disease in these patients, and there was limited information on available baseline characteristics of the patients and their tumors from the reports. In the meta-analyses, pooled estimates for the absolute frequency of changes from positive to negative ranged from $5.7 \%$ to $9.5 \%$ for ER status and $17 \%$ to $24 \%$ for PR status (Data Supplement Table 4). Pooled estimates for the absolute frequency of change from negative to positive ranged from $3 \%$ to $8.8 \%$ for ER status and from $6.9 \%$ to $7.3 \%$ for PR status. Pooled estimates were approximately $5.5 \%$ overall for the frequency of absolute change in HER2 status (in either direction). The meta-analyses did not report outcomes of systemic therapy for metastatic breast cancer.

For the seven individual studies, data on the frequency of change in receptor status did not differ substantially from the pooled estimates reported by the meta-analyses (Data Supplement Table 4). Of the seven available individual studies, one reported no significant difference in outcomes between patients with concordant versus discordant results for receptor status, ${ }^{18}$ whereas four reported shorter survival for those with specific discordances. ${ }^{20-24}$ However, none compared outcomes for patients with receptor discordances whose systemic therapy for metastatic disease was guided by results from the metastases versus results from the primary tumor. Thus, evidence is lacking to determine the optimal basis for selecting a treatment regimen when discordances are found.

\section{Clinical Interpretation}

The differential diagnosis for a lesion suggestive of a metastatic focus ranges from benign to malignant, including a new malignancy. Hence, when possible, this Panel recommends a biopsy be performed. If metastatic disease is confirmed, subsequent treatment planning can be made with confidence of the condition at hand. The Expert Panel reviewed the available data and found that the frequency of change from the primary tumor to the metastasis for ER, PR, or HER2 was uncommon, $<10 \%$ in all cases of pooled estimates. This represents a significant number of patients and is therefore clinically meaningful. There are several potential explanations for these changes, including 
variability in assay performance, tumor heterogeneity, and biologic evolution of the tumor. Although each reason for change may have its own potential significance, performing rebiopsy and retesting for ER, PR, and HER2 was believed to be justified in all cases, given that the choice of current therapy is largely determined by these biomarkers. The Panel believed that special circumstances should be noted in patients with bone metastases for whom results of ER, PR, and HER2 may be unreliable with current pathology practices.

The decalcification of bone biopsy material for the analysis of ER, PR, and HER2 may alter the outcome of the tumor analysis. ${ }^{33,34}$ Whenever possible, testing of bone metastases should be performed using a validated assay with a sample that has not been decalcified during preanalytic processing. Using ethylenedi-aminetetra-acetic acid-based decalcification solutions and trying to limit the time in formic acid decalcification represent possible options; however, both approaches have disadvantages and should be validated before being offered clinically for breast cancer biomarker analysis. ${ }^{34}$ The ability to physically separate viable tumor from host skeletal tissue, so that at least some of the tumor can be processed without having to undergo decalcification, would be clearly advantageous, but may not be practical on small-needle biopsy specimens. For needle biopsies of metastatic breast cancer to bone, submitting the accompanying blood clot in the specimen container separately from the fragments of bony tissue may yield viable tumor that would not have to undergo decalcification and could be used for the analysis of HER2 in breast cancer metastatic to skeletal tissue. If it is not possible to test a sample that has not undergone decalcification, a negative result should be interpreted with caution, and consideration should be given to an additional and more suitable biopsy sample for testing.

In cases of discordance between the primary and metastatic site, the clinician should ensure that appropriate assay procedures were performed, given that this has the potential to affect treatment of the patient. Finally, emerging evidence suggests a greater importance of both inter- and intratumoral heterogeneity than previously appreciated. Studies have shown that different metastatic sites may contain different biomarker profiles, and clones within tumors may vary by molecular profile. ${ }^{35-37}$ The clinical consequences of these findings are as yet unknown. The Panel informal consensus for the management of care when there is discordance of ER, PR, or HER2 results between primary and metastatic tissues is to use the ER, PR, or HER2 status from the metastasis to direct therapy, if supported by the clinical scenario and the patient's goals for care. Clinical judgment must be exercised.

The literature review and clinical interpretation for question 1 apply to all tumor subtypes: ER positive, PR positive, HER2 positive, and triple negative.

\section{CLINICAL QUESTION 2}

For women with metastatic breast cancer and with known endocrine receptor and HER2 status, which additional tumor markers have demonstrated clinical utility to initiate systemic therapy or direct selection of a new systemic therapy regimen?

\section{Recommendation 2}

Decisions concerning the initiation of systemic therapy or selection of systemic therapy for metastatic breast cancer should be guided by ER, PR, and HER2 status, in conjunction with clinical evaluation, judgment, and the patient's goals for care. Presently, there is no evidence that therapy decisions based solely on additional biomarker results improve health outcomes; thus, decisions about initiating or selecting therapy for metastatic breast cancer should be based solely on ER, PR, and HER2 status and the specific clinical scenario. (Type: evidence based. Evidence quality: low. Strength of recommendation: moderate.)

\section{Literature Review and Analysis}

Five articles reporting on the results of prospective-retrospective studies investigated the use of biomarker results to initiate treatment for metastatic breast cancer. Di Leo et $\mathrm{al}^{28}$ evaluated the role of $p 53$ gene mutations in predicting response to doxorubicin versus docetaxel, with no evidence of differential response on the basis of the biomarker. Finn et $\mathrm{al}^{29}$ evaluated the role of epidermal growth factor receptor expression in predicting response to lapatinib, a tyrosine kinase inhibitor, in a trial of paclitaxel with or without lapatinib. No interaction between epidermal growth factor receptor and response to lapatinib was found. Galmarini et $\mathrm{al}^{30}$ evaluated microtubuleassociated proteins (total $\alpha$ - and $\beta$-tubulin, class II to IV $\beta$-tubulin isotopes, $\tau$ protein) and response to docetaxel versus doxorubicin, with differences in response rate; however, no time-to-progression or overall survival data were reported. Kroger et $\mathrm{al}^{31}$ evaluated Ki-67, p53, and BCL-2 in high- versus standard-dose therapy and reported a significant improvement in progression-free survival in patients with p53 wild type. Finally, Miles et $\mathrm{al}^{32}$ evaluated vascular endothelial growth factor A, E-selectin, vascular endothelial growth factor receptors 1 and 2, intracellular adhesion molecule 1 , and vascular cell adhesion molecule 1 in patients treated with docetaxel with or without bevacizumab; however, none of these markers was predictive of benefit from bevacizumab. Although the designs were sound, none of these studies established the clinical utility of the markers evaluated for selection of therapy for patients with metastatic breast cancer.

Next-generation sequencing of tumors has not demonstrated clinical utility, as defined in this guideline. The Panel does not recommend use of next-generation sequencing of the tumor to initiate systemic therapy or direct selection of a new systemic therapy regimen outside of a research setting. However, the Panel notes that therapeutic clinical trials investigating specific pathways are ongoing, and study eligibility may require documentation of a specific genetic profile. This scenario is considered investigational and warrants detailed discussion with the patient about the lack of clinical utility of next-generation sequencing.

\section{Clinical Interpretation}

The consensus of the expert Panel supports the use of ER, PR, and HER2 to guide practice, as outlined in the recent updated ASCO guideline on HER2 testing ${ }^{3}$ and on ER and PR testing. ${ }^{4}$ No additional biomarkers for clinical use are recommended outside of a clinical trial.

The literature review and clinical interpretation for question 2 apply to all tumor subtypes: ER positive, PR positive, HER2 positive, and triple negative.

\section{CLINICAL QUESTION 3}

For women with metastatic breast cancer and with known ER, PR, and HER2 status, which additional tumor markers have demonstrated clinical utility to guide decisions on switching to a different drug or regimen or discontinuing treatment? 


\section{Recommendation 3}

Recommendations for tissue biomarkers. In patients who are already receiving systemic therapy for metastatic breast cancer, decisions on changing to a new drug or regimen or discontinuing treatment should be based on the patient's goals for care and clinical evaluation and judgment of disease progression or response, given that there is no evidence at this time that changing therapy solely on the basis of biomarker results beyond ER, PR, and HER2 improves health outcome, quality of life, or cost effectiveness. (Type: evidence based. Evidence quality: low. Strength of recommendation: moderate.)

Recommendations for circulating tumor markers. (Note: One author [D.F.H.] recused himself from deliberations and Panel votes concerning recommendations for CTCs because of potential conflicts of interest.) In patients already receiving systemic therapy for metastatic breast cancer, decisions on changing to a new drug or regimen or discontinuing treatment should be based on the patient's goals for care and clinical evaluation and judgment of disease progression or response, given that there is no evidence at this time that changing therapy solely on the basis of circulating biomarker results improves health outcome, quality of life, or cost effectiveness. (Type: evidence based. Evidence quality: intermediate. Strength of recommendation: moderate.)

Carcinoembryonic antigen (CEA), cancer antigen (CA) 15-3, and CA 27-29 may be used as adjunctive assessments to contribute to decisions regarding therapy for metastatic breast cancer. Data are insufficient to recommend use of CEA, CA 15-3, and CA 27-29 alone for monitoring response to treatment. The Panel acknowledges the lack of evidence of clinical utility in support of use of these circulating biomarkers; biochemical assessments of CEA, CA 15-3, and CA 27-29 were developed before the present standards for measuring clinical utility. The recommendation for use is based on clinical experience and Panel informal consensus in the absence of studies designed to evaluate the clinical utility of the markers. As such, it is also reasonable for clinicians to not use these markers as adjunctive assessments. (Type: informal consensus. Evidence quality: insufficient. Strength of recommendation: moderate.)

\section{Literature Review and Analysis}

There were no studies that met eligibility criteria for circulating markers to guide the changing or discontinuation of treatment. A number of studies have evaluated the role of CTCs in metastatic disease and have clearly shown that CTCs are associated with poor prognosis in this setting. However, none of the reviewed studies assessed predictive value using clinical utility guidelines, with one exception. The only study found to address markers used to discontinue or change therapy reported the results of a randomized trial of change in CTC level to decide whether to change or continue a treatment regimen after one cycle of first-line chemotherapy (of physician's choice) for metastatic breast cancer ${ }^{27}$ (Data Supplement Table 5). Investigators measured CTC levels for patients about to start first-line chemotherapy for metastatic breast cancer. Comparison of progression-free and overall survival for study arms confirmed previously reported data $^{38}$ showing that CTC level $\geq 5 / 7.5 \mathrm{~mL}$ of blood is a prognostic marker. However, comparison of progression-free and overall survival showed no difference in outcome when patients were switched to an alternate regimen on the basis of CTC level. These data also illustrate the frequently observed result that biomarkers may be prognostic but not predictive for clinical benefit when used to guide or influence decisions on systemic therapy for metastatic breast cancer.

Clinical experience and Panel informal consensus support the 2007 ASCO biomarker guideline ${ }^{1}$ that serum markers (CEA, CA 15-3, CA 27-29) may provide an adjunct decision-making tool in the metastatic setting, but should not be used as a standalone tool. As noted in the 1996 ASCO biomarker guideline, ${ }^{39}$ in the absence of readily measurable disease, increasing CEA, CA 15-3, or CA 27-29 may be used to indicate treatment failure. If monitoring of levels is performed, it should only be performed in patients with documented elevation, and a $20 \%$ to $30 \%$ change is required, along with supporting clinical evidence, before considering discontinuation of therapy. ${ }^{39}$ Caution should be used when interpreting increasing CEA, CA 15-3, or CA 27-29 level during the first 4 to 6 weeks of administration of a new therapy, given that spurious early increases may occur. The clinical validity of these markers, coupled with the clinical experience of Panel members, led to the informal consensus to support the use of serial CEA, CA 15-3, and CA 27-29 as adjunctive assessments to aid in clinical decisions. Not one of the circulating markers has shown clinical utility—by contemporary standards- to support its use as an independent predictive marker.

\section{Clinical Interpretation}

The Panel reviewed the available data on markers evaluated to guide decisions to discontinue or change therapy for metastatic breast cancer and agrees there is no evidence for clinical utility, as defined by the contemporary definition (ie, change in treatment based on the marker is shown to improve patient outcome in a rigorous fashion).

The literature review and clinical interpretation for question 3 apply to all tumor subtypes: ER positive, PR positive, HER2 positive, and triple negative.

\section{CLINICAL QUESTION 4}

For biomarkers shown to have clinical utility to guide decisions on systemic therapy for metastatic disease in questions 2 and 3, what are the appropriate assays, timing, and frequency of measurement?

\section{Recommendation 4}

Decisions for systemic therapy should be influenced by ER, PR, and HER2. ASCO recently updated the guideline addressing optimization of HER2 assays. ${ }^{3}$ To date, clinical utility has not been demonstrated for any additional biomarkers. (Type: informal consensus. Evidence quality: low. Strength of recommendation: strong.)

\section{Literature Review and Analysis}

No studies have demonstrated clinical utility beyond ER, PR, and HER2; the ASCO guidelines for these biomarkers are available at: http:// www.instituteforquality.org/recommendations-human-epidermalgrowth-factor-receptor-2-testing-breast-cancer-american-society and http://www.instituteforquality.org/asco-cap-guidelinerecommendations-immunohistochemical-testing-estrogen-andprogesterone-receptors. Given the special circumstances surrounding CEA, CA 15-3, and CA 27-29, if a clinician chooses to use these serum markers to aid in treatment decisions, these tests should be performed in compliance with previously published ASCO guidelines. ${ }^{39}$

\section{Clinical Interpretation}

The Panel recommends that outside of clinical trials, the process of clinical decision making should be informed by ER, PR, and 
HER2. Although other biomarkers are in active development, there is insufficient evidence to demonstrate that their use improves cancer outcomes.

The literature review and clinical interpretation for question 4 apply to all tumor subtypes: ER positive, PR positive, HER2 positive, and triple negative.

\section{PATIENT AND CLINICIAN COMMUNICATION}

Patients (and family members or caregivers) should be educated about the results of pathology tests and how they are used to develop treatment plans tailored to the biology of patients' cancers. Because many newly diagnosed patients are under emotional stress and/or may be unaccustomed to complex medical terminology, the use of easily understood language (at an educational level that the patient can understand) is key to clear communication. Asking patients to repeat key pieces of information, providing written or recorded notes, and using visual aids can help ensure that information is effectively communicated. Patients should be given a copy of their pathology report and ER, PR, and HER2 test results. The clinician should review the results with the patient, discuss any issues with the test interpretation or performance, and ask if the patient has any additional questions about the results.

Appendix Table A2 (online only), adapted from the guideline update by Wolff et $\mathrm{al}^{3}$ offers clinicians a set of discussion points for communicating about the use of biomarkers to guide therapy decisions for metastatic breast cancer.

\section{HEALTH DISPARITIES}

Although ASCO clinical practice guidelines represent expert recommendations on the best practices in disease management to provide the highest level of cancer care, it is important to note that many patients have limited access to medical care. Racial and ethnic disparities in health care contribute significantly to this problem in the United States. Patients with cancer who are members of racial/ethnic minorities disproportionately experience comorbidities, experience more substantial obstacles to receiving care, are more likely to be uninsured, and are at greater risk of receiving poor-quality care than other Americans. ${ }^{18,40-42}$ Many other patients lack access to care because of their geographic location and distance from appropriate treatment facilities. Awareness of these disparities in access to care should be considered in the context of this clinical practice guideline, and health care providers should strive to deliver the highest level of cancer care to these vulnerable populations.

The biomarker distribution of ER, PR, and HER2 status can vary across tumors in patients with differing ethnic and racial backgrounds. ${ }^{43}$ In addition to the biologic variability of ER, PR, and HER2 expression within breast cancer, there is evidence that disparities exist in the frequency of biomarker testing in certain populations ${ }^{44}$ and that these disparities are influenced by health insurance coverage. ${ }^{45}$ The literature search performed for this guideline did not identify studies that examined the clinical utility of biomarkers across ethnic, racial, or socioeconomic backgrounds.

\section{MULTIPLE CHRONIC CONDITIONS}

Creating evidence-based recommendations to inform treatment of patients with additional chronic conditions, a situation in which the patient may have $\geq$ two such conditions-referred to as multiple chronic conditions (MCCs) - is challenging. Patients with MCCs are a complex and heterogeneous population, making it difficult to account for all of the possible permutations to develop specific recommendations for care. In addition, the best available evidence for treating index conditions, such as cancer, is often from clinical trials, the study selection criteria of which may exclude these patients to avoid potential interaction effects or confounding of results associated with MCCs. As a result, the reliability of outcome data from these studies may be limited, thereby creating constraints for expert groups to make recommendations for care in this heterogeneous patient population.

There have been reports of liver and renal dysfunction affecting the values of serum tumor markers, ${ }^{46,47}$ which demonstrates that MCCs may confuse the interpretation of serum tumor markers. There is insufficient evidence to calibrate how MCCs may affect the results of biomarker testing.

Given that many patients to whom guideline recommendations apply may present with MCCs, any treatment plan needs to take into account the complexity and uncertainty created by the presence of MCCs and highlight the importance of shared decision making with respect to guideline use and implementation. Therefore, in consideration of recommended care for the target index condition, the clinician should review all other chronic conditions present in the patient and take those conditions into account when formulating the treatment and follow-up plans.

In light of these considerations, practice guidelines should provide information on how to apply the recommendations for patients with MCCs, perhaps as a qualifying statement for recommended care. This may mean that some or all of the recommended care options are modified or not applied, as determined by best practice in consideration of any MCC.

\section{GUIDELINE IMPLEMENTATION}

ASCO guidelines are developed for implementation across health settings. Barriers to implementation include the need to increase awareness of the guideline recommendations among front-line practitioners and survivors of cancer and caregivers and also to provide adequate services in the face of limited resources. The guideline Bottom Line Box was designed to facilitate implementation of recommendations. This guideline will be distributed widely through the ASCO Practice Guideline Implementation Network. ASCO guidelines are posted on the ASCO Web site and usually published in Journal of Clinical Oncology and Journal of Oncology Practice.

\section{LIMITATIONS OF THE RESEARCH}

The research on predictive biomarkers in metastatic breast cancer is limited by the lack of prospective confirmatory studies, limited clinical utility, and, in many cases, the lack of clinical validity and reproducibility of the assays available for use. The Expert Panel awaits the 
completion and publication of ongoing and completed randomized trials to confirm and demonstrate the clinical utility of some of these assays. Extensive research is needed to identify and validate some of the biomarker candidates described.

ASCO believes that cancer clinical trials are vital to inform medical decisions and improve cancer care and that all patients should have the opportunity to participate.

\section{FUTURE DIRECTIONS}

One of the most important conclusions from this guideline is the compelling need for further research on this topic. With the substantial advances in treatment of women with advanced disease, including improved survival in metastatic breast cancer ${ }^{48}$ that is thought to be partly a result of improved targeted therapy with drugs such as trastuzumab, motivation for identifying robust biomarkers of response to therapy is clear. However, the study of biomarkers continues to lag behind that of the therapies themselves, because historically, this has not been an area of robust research. The reasons are multifactorial and include lack of a clear framework for conducting biomarker studies, lack of substantial investment by pharmaceutical companies and academic centers, and challenges in identifying cohorts of samples that could be adequately studied. However, these issues are changing, as exemplified by excellent publications on how to conduct biomarker research that will show robust evidence of patient impact, ${ }^{10,11,13,49}$ increased interest by pharmaceutical companies in so-called compan- ion diagnostics, and an increase in funding mechanisms by the $\mathrm{Na}$ tional Cancer Institute, emphasizing the importance of this kind of work. In this era of molecular medicine and patient-centered care, it is critical that the medical community continue to lobby for and conduct high-quality biomarker research for women with advanced breast cancer.

\section{ADDITIONAL RESOURCES}

More information, including a Data Supplement with additional evidence tables, a Methodology Supplement with information about evidence quality and strength of recommendations, slide sets, and clinical tools and resources, is available at http://www.asco.org/guidelines/ metastaticbreastmarkers. Patient information is available at http:// www.cancer.net.

\section{AUTHORS' DISCLOSURES OF POTENTIAL CONFLICTS OF INTEREST}

Disclosures provided by the authors are available with this article at www.jco.org.

\section{AUTHOR CONTRIBUTIONS}

Manuscript writing: All authors

Final approval of manuscript: All authors

\section{REFERENCES}

1. Harris L, Fritsche $H$, Mennel $R$, et al: American Society of Clinical Oncology 2007 update of recommendations for the use of tumor markers in breast cancer. J Clin Oncol 25:5287-5312, 2007

2. Wolff AC, Hammond ME, Schwartz JN, et al: American Society of Clinical Oncology/College of American Pathologists guideline recommendations for human epidermal growth factor receptor 2 testing in breast cancer. J Clin Oncol 25:118-145, 2007

3. Wolff AC, Hammond ME, Hicks DG, et al: Recommendations for human epidermal growth factor receptor 2 testing in breast cancer: American Society of Clinical Oncology/College of American Pathologists clinical practice guideline update. J Clin Oncol 31:3997-4013, 2013

4. Hammond ME, Hayes DF, Dowsett M, et al: American Society of Clinical Oncology/College of American Pathologists guideline recommendations for immunohistochemical testing of estrogen and progesterone receptors in breast cancer. J Clin Oncol 28:2784-2795, 2010

5. Ginsburg GS, Kuderer NM: Comparative effectiveness research, genomics-enabled personalized medicine, and rapid learning health care: A common bond. J Clin Oncol 30:4233-4242, 2012

6. Hayes DF, Allen J, Compton C, et al: Breaking a vicious cycle. Sci Transl Med 5:196cm6, 2013

7. Janes H, Pepe MS, Bossuyt PM, et al: Measuring the performance of markers for guiding treatment decisions. Ann Intern Med 154:253-259, 2011

8. McShane LM, Hayes DF: Publication of tumor marker research results: The necessity for complete and transparent reporting. J Clin Oncol 30:42234232, 2012
9. Kaufmann M, Pusztai L: Use of standard markers and incorporation of molecular markers into breast cancer therapy: Consensus recommendations from an international expert panel. Cancer 117:1575-1582, 2011

10. Polley MY, Freidlin B, Korn EL, et al: Statistical and practical considerations for clinical evaluation of predictive biomarkers. J Natl Cancer Inst 105:16771683, 2013

11. Simon RM, Paik S, Hayes DF: Use of archived specimens in evaluation of prognostic and predictive biomarkers. J Natl Cancer Inst 101:1446-1452, 2009

12. Hayes DF, Bast RC, Desch CE, et al: Tumor marker utility grading system: A framework to evaluate clinical utility of tumor markers. J Natl Cancer Inst 88:1456-1466, 1996

13. Teutsch SM, Bradley LA, Palomaki GE, et al: The Evaluation of Genomic Applications in Practice and Prevention (EGAPP) initiative: Methods of the EGAPP Working Group. Genet Med 11:3-14, 2009

14. Botkin JR, Teutsch SM, Kaye $\mathrm{Cl}$, et al: Outcomes of interest in evidence-based evaluations of genetic tests. Genet Med 12:228-235, 2010

15. Shiffman RN, Michel G, Rosenfeld RM, et al: Building better guidelines with BRIDGE-Wiz: Development and evaluation of a software assistant to promote clarity, transparency, and implementability. J Am Med Inform Assoc 19:94-101, 2012

16. Houssami N, Macaskill $P$, Balleine $R L$, et al: HER2 discordance between primary breast cancer and its paired metastasis: Tumor biology or test artefact? Insights through meta-analysis. Breast Cancer Res Treat 129:659-674, 2011

17. Zhang L, Riethdorf S, Wu G, et al: Metaanalysis of the prognostic value of circulating tumor cells in breast cancer. Clin Cancer Res 18:57015710, 2012
18. Amir E, Clemons M, Purdie CA, et al: Tissue confirmation of disease recurrence in breast cancer patients: Pooled analysis of multi-centre, multidisciplinary prospective studies. Cancer Treat Rev 38:708-714, 2012

19. Amir E, Miller N, Geddie W, et al: Prospective study evaluating the impact of tissue confirmation of metastatic disease in patients with breast cancer. $\mathrm{J}$ Clin Oncol 30:587-592, 2012

20. Dieci MV, Barbieri E, Piacentini F, et al: Discordance in receptor status between primary and recurrent breast cancer has a prognostic impact: $A$ single-institution analysis. Ann Oncol 24:101-108, 2013

21. Hoefnagel LD, van de Vijver MJ, van Slooten $\mathrm{HJ}$, et al: Receptor conversion in distant breast cancer metastases. Breast Cancer Res 12:R75, 2010

22. Hoefnagel LD, Moelans CB, Meijer SL, et al: Prognostic value of estrogen receptor alpha and progesterone receptor conversion in distant breast cancer metastases. Cancer 118:4929-4935, 2012

23. Lindström LS, Karlsson E, Wilking UM, et al: Clinically used breast cancer markers such as estrogen receptor, progesterone receptor, and human epidermal growth factor receptor 2 are unstable throughout tumor progression. J Clin Oncol 30: 2601-2608, 2012

24. Niikura N, Liu J, Hayashi $N$, et al: Loss of human epidermal growth factor receptor 2 (HER2) expression in metastatic sites of HER2-overexpressing primary breast tumors. J Clin Oncol 30:593-599, 2012

25. Simmons C, Miller N, Geddie W, et al: Does confirmatory tumor biopsy alter the management of breast cancer patients with distant metastases? Ann Oncol 20:1499-1504, 2009 
26. Thompson AM, Jordan LB, Quinlan $P$, et al: Prospective comparison of switches in biomarker status between primary and recurrent breast cancer: The Breast Recurrence In Tissues Study (BRITS). Breast Cancer Res 12:R92, 2010

27. Smerage JB, Barlow WE, Hortobagyi GN, et al: Circulating tumor cells and response to chemotherapy in metastatic breast cancer: SWOG S0500. J Clin Oncol 32:3483-3489, 2014

28. Di Leo A, Tanner M, Desmedt C, et al: P-53 gene mutations as a predictive marker in a population of advanced breast cancer patients randomly treated with doxorubicin or docetaxel in the context of a phase III clinical trial. Ann Oncol 18:997-1003, 2007

29. Finn RS, Press MF, Dering J, et al: Estrogen receptor, progesterone receptor, human epidermal growth factor receptor 2 (HER2), and epidermal growth factor receptor expression and benefit from lapatinib in a randomized trial of paclitaxel with lapatinib or placebo as first-line treatment in HER2negative or unknown metastatic breast cancer. J Clin Oncol 27:3908-3915, 2009

30. Galmarini CM, Treilleux I, Cardoso F, et al: Class III beta-tubulin isotype predicts response in advanced breast cancer patients randomly treated either with single-agent doxorubicin or docetaxel. Clin Cancer Res 14:4511-4516, 2008

31. Kröger N, Milde-Langosch $K$, Riethdorf $S$, et al: Prognostic and predictive effects of immunohistochemical factors in high-risk primary breast cancer patients. Clin Cancer Res 12:159-168, 2006

32. Miles DW, de Haas SL, Dirix LY, et al: Biomarker results from the AVADO phase 3 trial of first-line bevacizumab plus docetaxel for HER2- negative metastatic breast cancer. $\mathrm{Br} \mathrm{J}$ Cancer 108:1052-1060, 2013

33. Darvishian F, Singh B, Krauter $S$, et al: Impact of decalcification on receptor status in breast cancer. Breast J 17:689-691, 2011

34. Alers JC, Krijtenburg PJ, Vissers KJ, et al: Effect of bone decalcification procedures on DNA in situ hybridization and comparative genomic hybridization: EDTA is highly preferable to a routinely used acid decalcifier. J Histochem Cytochem 47:703-710, 1999

35. Shipitsin M, Campbell LL, Argani $P$, et al: Molecular definition of breast tumor heterogeneity. Cancer Cell 11:259-273, 2007

36. Stoecklein NH, Klein CA: Genetic disparity between primary tumours, disseminated tumour cells, and manifest metastasis. Int $\mathrm{J}$ Cancer 126 : 589-598, 2010

37. Navin N, Kendall J, Troge J, et al: Tumour evolution inferred by single-cell sequencing. Nature 472:90-94, 2011

38. Cristofanilli $M$, Fortina $P$ : Circulating tumor DNA to monitor metastatic breast cancer. N Engl J Med 369:93, 2013

39. Clinical practice guidelines for the use of tumor markers in breast and colorectal cancer: Adopted on May 17, 1996 by the American Society of Clinical Oncology. J Clin Oncol 14:2843-2877, 1996

40. American Cancer Society: Cancer Facts and Figures for African Americans 2011-2012. Atlanta, GA, American Cancer Society, 2011

41. Howlader N, Noone AM, Krapcho M, et al: SEER Cancer Statistics Review, 1975-2009 (vintage 2009 populations). http://seer.cancer.gov/csr/ 1975_2009_pops09/
42. Mead $H$, Cartwright-Smith $L$, Jones $K$, et al: Racial and Ethnic Disparities in U.S. Health Care: A Chartbook. New York, NY, Commonwealth Fund, 2008

43. Singh $M$, Ding $Y$, Zhang $L Y$, et al: Distinct breast cancer subtypes in women with early-onset disease across races. Am J Cancer Res 4:337-352, 2014

44. Lund MJ, Mosunjac M, Davis KM, et al: 21-Gene recurrence scores: Racial differences in testing, scores, treatment, and outcome. Cancer 118:788-796, 2012

45. Stark A, Kucera G, Lu M, et al: Influence of health insurance status on inclusion of HER-2/neu testing in the diagnostic workup of breast cancer patients. Int J Qual Health Care 16:517-521, 2004

46. Estakhri R, Ghahramanzade A, Vahedi $A$, et al: Serum levels of CA15-3, AFP, CA19-9 and CEA tumor markers in cancer care and treatment of patients with impaired renal function on hemodialysis Asian Pac J Cancer Prev 14:1597-1599, 2013

47. Hayes DF, Zurawski VR Jr, Kufe DW: Comparison of circulating CA15-3 and carcinoembryonic antigen levels in patients with breast cancer. J Clin Oncol 4:1542-1550, 1986

48. Giordano SH, Temin S, Kirshner JJ, et al: Systemic therapy for patients with advanced human epidermal growth factor receptor 2-positive breast cancer: American Society of Clinical Oncology clinical practice guideline. J Clin Oncol 32:2078-2099, 2014

49. in Micheel CM, Nass SJ, Omenn GS (eds): Evolution of Translational Omics: Lessons Learned and the Path Forward. Washington, DC, National Academies Press, 2012 


\section{AUTHORS' DISCLOSURES OF POTENTIAL CONFLICTS OF INTEREST}

Use of Biomarkers to Guide Decisions on Systemic Therapy for Women With Metastatic Breast Cancer: American Society of Clinical Oncology Clinical Practice Guideline

The following represents disclosure information provided by authors of this manuscript. All relationships are considered compensated. Relationships are self-held unless noted. I = Immediate Family Member, Inst = My Institution. Relationships may not relate to the subject matter of this manuscript. For more information about ASCO's conflict of interest policy, please refer to www.asco.org/rwc or jco.ascopubs.org/site/ifc.

\section{Catherine Van Poznak}

Research Funding: Amgen (Inst), Novartis (Inst)

Patents, Royalties, Other Intellectual Property: UpToDate

\section{Mark R. Somerfield}

No relationship to disclose

Robert C. Bast

Research Funding: Arrian Pharmaceuticals

Patents, Royalties, Other Intellectual Property: Fujirebio Diagnostics

\section{Massimo Cristofanilli}

Honoraria: Agendia, Dompe, Cynvenio, NanoString Technologies

Consulting or Advisory Role: Dompe, Cynvenio, Newomics

Speakers' Bureau: Agendia, NanoString Technologies

\section{Elizabeth G. Hill}

Consulting or Advisory Role: Apogee Biotechnology Corporation

Matthew P. Goetz

Consulting or Advisory Role: Eli Lilly (Inst)

Travel, Accommodations, Expenses: Eli Lilly

Ana M. Gonzalez-Angulo

No relationship to disclose

\section{David G. Hicks}

Honoraria: Genentech

Speakers' Bureau: Genentech

Travel, Accommodations, Expenses: Genentech

Minetta C. Liu

Research Funding: Eisai (Inst), Seattle Genetics (Inst), Celgene (Inst), Veridex (Inst), Clearbridge Biomedics (Inst), Novartis (Inst),

Roche/Genentech (Inst)

\section{Wanda Lucas}

No relationship to disclose
Ingrid A. Mayer

Consulting or Advisory Role: Novartis, Genentech/Roche, Clovis Research Funding: Novartis

Robert G. Mennel

Employment: Texas Oncology, Baylor Health Care System Stock or Other Ownership: MedFusion

William F. Symmans

Stock or Other Ownership: Amgen, ISIS Pharmaceuticals, Nuvera Bioiences

Honoraria: Orlando Health

Patents, Royalties, Other Intellectual Property: Patent for the formula to calculate residual cancer burden after neoadjuvant chemotherapy; patent pending for genomic signatures of estrogen receptor-related gene expression and for prediction of response and survival from adjuvant chemotherapy and/or endocrine therapy

Travel, Accommodations, Expenses: Affymetrix, Celgene

Daniel F. Hayes

Stock or Other Ownership: Oncimmune, Inbiomotion Honoraria: Third UK Breast Cancer Meeting, London, Lilly Oncology Consulting or Advisory Role: Pfizer

Research Funding: Janssen R\&D (Johnson \& Johnson parent company) (Inst), AstraZeneca (Inst), Puma Biotechnology (Inst), Pfizer (Inst) Patents, Royalties, Other Intellectual Property: Title: A method for predicting progression free and overall survival at each follow-up timepoint during therapy of metastatic breast cancer patients using circulating tumor cells; patent No. 05725638.0-1223-US2005008602; Title: Diagnosis and treatment of breast cancer; original application No. 61/079, 642; revised application No. 61/224, 310; Title: Circulating tumor cell capturing techniques and devices; original application No. 61/593, 092; royalties from licensed technology

Lyndsay N. Harris

No relationship to disclose 


\section{Acknowledgment}

We thank the Clinical Practice Guideline Committee for its thoughtful review of and insightful comments on this guideline document and Kari Bohlke, ScD, and Nofisat Ismaila, MD, for assistance with data extraction.

\section{Appendix}

\begin{tabular}{|ll|}
\hline \multicolumn{1}{|c|}{ Member } & Table A1. Panel Members \\
\hline Lyndsay N. Harris, MD (co-chair) & \\
Catherine Van Poznak, MD (co-chair, PGIN representative) & Seidman Cancer Center, Case Western Reserve University, Cleveland, OH \\
Robert C. Bast, MD & University of Michigan Comprehensive Cancer Center, Ann Arbor, MI \\
Massimo Cristofanilli, MD & University of Texas MD Anderson Cancer Center, Houston, TX \\
Elizabeth G. Hill, PhD & Thomas Jefferson University-Kimmel Cancer Center, Philadelphia, PA \\
Matthew P. Goetz, MD & Medical University of South Carolina, Hollings Cancer Center, Charleston, SC \\
Daniel F. Hayes, MD & Mayo Clinic, Rochester, MN \\
David G. Hicks, MD & University of Michigan Comprehensive Cancer Center, Ann Arbor, MI \\
Minetta C. Liu, MD & University of Rochester Medical Center, Rochester, NY \\
Wanda Lucas, MBA (patient representative) & Mayo Clinic, Rochester, MN \\
Ingrid A. Mayer, MD, MSCl & Georgetown University, Washington, DC \\
Robert G. Mennel, MD & Vanderbilt University Medical Center and Vanderbilt-Ingram Cancer Center, Nashville, TN \\
William F. Symmans, MD & Texas Oncology, Dallas, TX \\
\hline Abbreviation: PGIN, Practice Guideline Implementation Network. & University of Texas MD Anderson Cancer Center, Houston, TX \\
\hline
\end{tabular}

Table A2. Suggested Discussion Points Between Patient and Medical Provider on Use of Biomarkers to Guide Therapy Decisions for Metastatic Breast Cancer

$$
\text { Preamble }
$$

Establish how patient prefers to receive information and patient's perceptions of his or her diagnosis as well as tumor factors that might influence decision making

Educate patient (and family members or caregivers) about results of tests and how they are used to develop treatment plan tailored to biology of cancer Use of easily understood language (at educational level patient can understand) is key to clear communication

$$
\text { Key Point Action }
$$

Explain importance of determining Patients should understand that most common biologic tests are for ER, PR, and HER2 and that testing for these markers biologic characteristics of is important to select appropriate treatment; patients should be informed about which tests were performed and breast cancer expected turnaround time for these tests

Patients and clinicians should understand that wide range of biomarker tests are technically possible, but only ER, PR, and HER2 have demonstrated clinical validity for metastatic breast cancer

Patients should understand that assay validity and clinical validity of biomarker are important steps in test development but by themselves are insufficient to guide treatment decisions outside of clinical trial

Explain importance of ER, PR, Patients should understand that ER, PR, and HER2 status determines whether certain drugs (eg, endocrine therapy, and HER2 testing trastuzumab, lapatinib, pertuzumab, T-DM1) are recommended; they should have understanding of means by which markers serve as prognostic and predictive factors

Patient should be informed that Panel informal consensus for management of care when there is discordance of ER, PR or HER2 results between primary and metastatic tissues is to use ER, PR, or HER2 status from metastasis to direct therapy, if supported by clinical scenario and patient's goals for care

Explain types of tests used to determine ER, PR, and HER2 status

Patients should understand that there are different FDA-approved testing methods that detect ER, PR, and HER2 status, including evaluation of protein overexpression and presence of genetic alterations (eg, gene alterations)

Guidelines are in place for testing biomarkers on tumor specimens; patients may be referred to ASCO/CAP guideline update on ASCO or CAP Web site for additional patient-focused information

Explain interpretation of ER, PR, Patients should understand that although most test results are definitively positive or negative, there are equivocal results and HER2 test results that require additional testing using alternative test or using same or alternative test on different portion of same specimen (different block); oncologist or pathologist may recommend additional testing using different type of tumor specimen (eg, surgical excision $v$ core biopsy), if available

Unfortunately, some results remain indeterminate or inconsistent with other histopathologic findings; in such cases, final treatment decision to consider treatment with ER-, PR-, or HER2-targeted therapy should be made after consultation between pathologist and oncologist and discussion with patient

Explain importance of retesting $E R, P R$, and HER2 status in new, metastatic tumors

Patients should understand that ER, PR, and HER2 status may occasionally be different (discordant) when comparing previous primary tumor and site of recurrence or in setting of multiple simultaneous metastatic sites; in some cases, it is not possible to fully differentiate between true biologic change, tumor heterogeneity, or variability in performance of assay

NOTE. Adapted with permission. ${ }^{3}$

Abbreviations: ASCO, American Society of Clinical Oncology; CAP, College of American Pathologists; ER, estrogen receptor; FDA, US Food and Drug Administration; HER2, human epidermal growth factor receptor 2; PR, progesterone receptor; T-DM1, trastuzumab emtansine. 